\title{
Partial Height Harmony as Partial Transparency
}

\author{
Caitlin Smith \\ Johns Hopkins University
}

\section{Introduction}

Harmony can be defined broadly as the spread of some phonological property from a trigger segment to one or more undergoer segments within some domain. Transparency to harmony refers to a situation in which some segments in a domain do not take on a harmonizing property, but instead have apparently been skipped over. There are also cases in which some segments appear to have only partially undergone harmony, assimilating to a trigger segment with respect to a harmonizing property, but not necessarily taking on that property completely.

Partial harmony is most commonly exemplified by cases of height harmony in which an undergoer vowel approaches the height of a trigger vowel without necessarily reaching it. One such partial height harmony is the bounded vowel raising harmony (metaphony) of Servigliano Italian (Camilli 1929, Kaze 1989, Nibert 1998, Mascaró 2011, Walker 2011), in which a high vowel in a suffix triggers raising of preceding mid vowels, up to and including the stressed vowel. The high-mid vowels /e/ and /o/ surface faithfully before non-high vowels, as in (1a-d). However, they surface as [i] and [u], respectively, before high vowels, as in (1e-h). (All data are from Camilli, with interpretation of the various harmony patterns in the language from Nibert and Walker.)
(1) a. [kréd-o] 'I believe'
b. [pés-a] 'heavy (f. sg.)'
c. [fjór-e] 'flower (m. sg.)'
d. [lóng-a] 'long (f. sg.)'
e. [kríd-i] 'you believe'
f. [pís-u] 'heavy (m. sg.)'
g. [fjúr -i] 'flower (m. pl.)'
h. [lúng-u] 'long (m. sg.)'

The low-mid vowels also undergo raising when followed by a high vowel, but do not surface as high vowels themselves. Underlying $/ \varepsilon /$ and $/ \mathrm{s} /$ surface faithfully before non-high vowels, as in (2a-d), and as high-mid [e] and [o], respectively, before high vowels, as in (2e-h).
(2) a. [pétten-e] 'comb (m. sg.)'

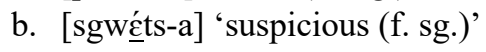
c. [mór-e] 'he dies'
d. [móf-a] 'dejected (f. sg.)'
e. [péttin-i] 'comb (m. pl.)'
f. [sgwéts-u] 'suspicious (m. sg.)'
g. [mór-i] 'you die'
h. [móf-u] 'dejected (m. sg.)'

Finally, the low vowel [a] occurs before vowels of any height, indicating that it does not undergo raising. This is illustrated by the data in (3).
(3) a. [pátr-e] 'father (m. sg.)'
c. [pátr-i] 'father (m. pl.)'
b. [kár r-a] 'dear (f. sg.)'
d. [kár r-u] 'dear (m. sg.)'

\footnotetext{
* For helpful discussion of this work I thank Louis Goldstein, Brian Hsu, Hayeun Jang, Andrew Lamont, Adam McCollum, Elliott Moreton, Charlie O’Hara, Amy Reynolds, Eric Rosen, Hannah Sande, Jennifer Smith, Paul Smolensky, Rachel Walker, Doug Whalen, and audiences at UNC Chapel Hill, Princeton University, Georgetown University, AMP 2019, NELS 50, and LSA 2020. This research is supported by Microsoft Research AI.
}

(C) 2020 Caitlin Smith

Proceedings of $A M P 2019$ 
The vowel raising pattern in Servigliano Italian is summarized in the figure in (4), which shows that this is a case of partial, stepwise height harmony. Underlying high-mid vowels raise to become high like the trigger vowel, while underlying low-mid vowels approach the trigger's height rather than taking it on completely.

(4) Vowel raising pattern in Servigliano Italian metaphony
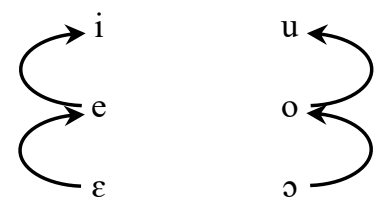

a

In this paper, I propose an analysis of Servigliano Italian metaphony that is situated within the Gestural Harmony Model (Smith 2016, 2017a, 2017b, 2018). In this model, the subsegmental units of phonological representation are gestures, the goal-based, dynamically-defined units that are assumed in the framework of Articulatory Phonology (Browman \& Goldstein 1986, 1989). Harmony is the result of a gesture extending to overlap the gestures of other segments in a word, and transparency arises due to overlap between a harmonizing gesture and a gesture with a conflicting articulatory target, resulting in competition for control of the vocal tract. In this model of harmony and transparency, I propose that cases in which segments seem to partially undergo harmony be analyzed as cases of partial transparency, resulting from competition between conflicting gestures of similar strengths. This includes cases of partial height harmony such as low-mid vowel raising in Servigliano Italian metaphony, which I analyze as a case of partial transparency to harmony.

The paper is organized as follows. Section 2 introduces gestures as units of phonological representation and outlines the Gestural Harmony Model. Section 3 presents the analysis of Servigliano Italian metaphony within that model. Section 4 discusses issues that arise in analyses of partial height harmony relying on featural representations and cumulative constraint interaction. Section 5 concludes.

\section{Representing Vowel Harmony with Gestures}

2.1 Gestures as phonological units In this paper, I adopt gestures as the units of phonological representation. Originally conceived within the framework of Articulatory Phonology (Browman \& Goldstein 1986, 1989), gestures are goal-based units that are specified for a target articulatory state. The achievement of that target articulatory state unfolds over time according to a dynamically-defined equation of motion. The length of time over which a gesture commands the vocal tract to achieve its target state is its activation period. When sufficient time has passed for its target state to be achieved, a gesture deactivates, and its articulators may return to specified neutral positions until they are recruited by subsequent gestures.

A gesture's target articulatory state is specified in terms of a primary articulator, constriction location, and constriction degree. The constriction location of a consonantal gesture is specified as some point along the static surface of the vocal tract. Constriction degree refers to how narrow or wide the constriction between the primary articulator and the constriction location is. This is illustrated in (5), which shows several possible constriction locations for a consonantal gesture of the tongue body. 
(5) Target constriction locations for consonantal gestures of the tongue body

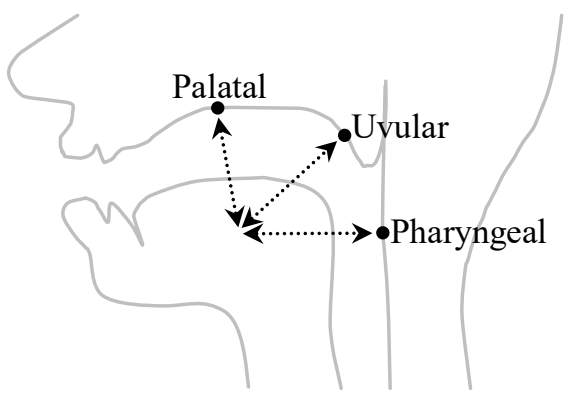

Often, the target constriction locations of vowel gestures are assumed to be specified along the same radial axis as consonantal gestures. However, this assumption does not admit direct encoding of vowel height and backness, dimensions along which vowels are highly phonologically active. Therefore, I diverge from the standard gestural representation of vowels here and propose a new set of gestures for vowels. According to this new representational scheme, vocalic gestures are still specified for constriction location and degree, just as consonantal gestures are. However, rather than specifying constriction location as a specific point along the vocal tract, I propose that vowels are composed of two tongue body gestures whose constriction locations are specified for fairly wide regions of the vocal tract. One of these gestures has a target constriction location comprising much of the upper surface of the vocal tract, including the palate and velum. The target articulatory state of this gesture is to make a constriction anywhere in this region. Its constriction degree determines a vowel's height; a narrow constriction at the upper surface produces a high vowel, while a wide constriction produces a low vowel. The other vowel gesture I propose has a target constriction location comprising much of the back surface of the vocal tract, including the uvula and pharynx. The constriction degree of a tongue body back surface constriction gesture determines a vowel's backness. A narrow constriction in this region produces a back vowel, while a wide constriction produces a front vowel. The target constriction locations for these newly proposed vowel gestural are illustrated in (6).

(6) Tongue body position tasks for upper surface and back surface gestures

(a) Tongue body upper surface gesture

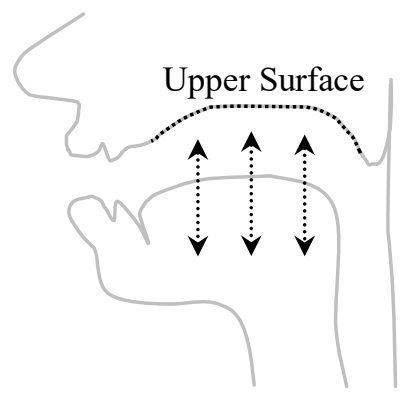

(b) Tongue body back surface gesture

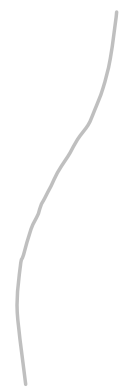

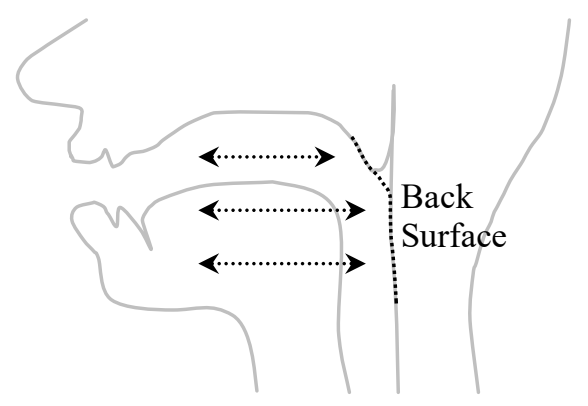

By adopting these new types of constriction location regions for vocalic gestures, vowel height and backness are encoded directly by gestural representations. This is crucial to outlining an analysis of height harmony within the Gestural Harmony Model, which is introduced in the next section.

2.2 The Gestural Harmony Model In the Gestural Harmony Model (Smith 2016, 2017a, 2017b, 2018), harmony results when a gesture extends its period of activation and overlaps the gestures of other segments in a domain. To achieve this extended gestural activation, Smith proposes that a gesture is specified as to whether or not it self-activates at its specified starting point and whether or not it selfdeactivates once it reaches its target articulatory state. These parameters determine whether a gesture is a trigger of harmony. 
The effects of these gestural parameter specifications are illustrated in (7). In (7a) is a typical selfactivating and self-deactivating lip protrusion gesture, which is used to represent rounding. When this gesture reaches its target articulatory state (protruded lips), it self-deactivates. The gesture in (7b) is a persistent, or non-self-deactivating gesture. The dashed line indicates the point at which the gesture reaches its target articulatory state. As a persistent gesture, it does not deactivate at this point, but instead remains active, extending and overlapping the gestures of following segments. The gesture in $(7 \mathrm{c})$ is an anticipatory, or early-activating gesture. The dashed line indicates the point at which the gesture is scheduled to start according to its position in a word. However, since it is an anticipatory gesture it has activated before that point, extending to overlap the gestures of preceding segments.

(7) Typical, persistent (non-self-deactivating), and anticipatory (early-activating) lip protrusion gestures

(a) Typical gesture

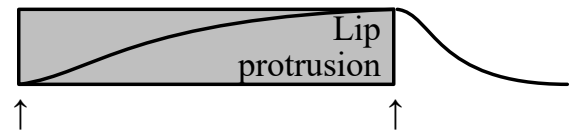

starting timepoint, target articulatory state reached, gesture activates gesture self-deactivates

(b) Persistent gesture

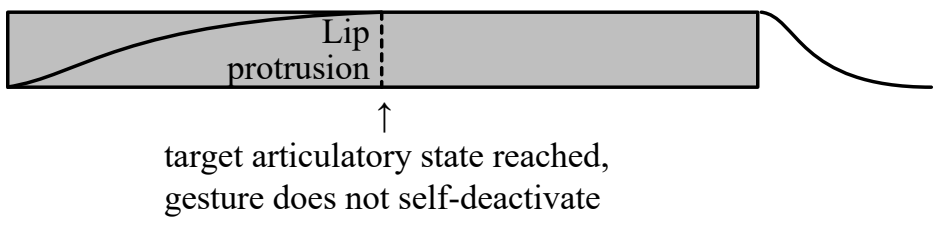

(c) Anticipatory gesture

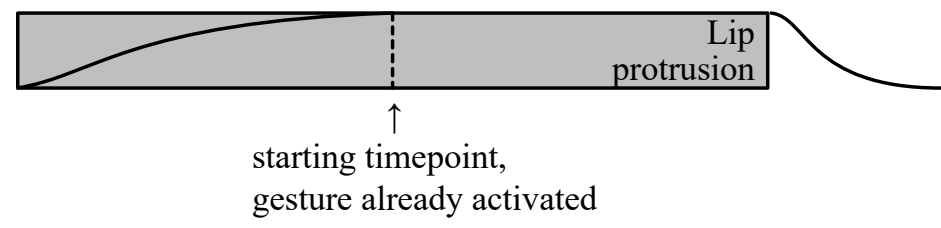

In the Gestural Harmony Model, harmony arises when a segment includes a gesture that is either persistent, anticipatory, or both; that segment is the trigger of harmony. Other segments undergo harmony when their composite gestures are overlapped by a harmonizing gesture.

The figure in (8) illustrates this with a gestural score for an [o-o] sequence, in which the first $[\mathrm{o}]$ is the trigger of harmony, and the second [o] is the undergoer. The segmental transcription is provided along the top, and the subscript for each segment matches the subscripts of its composite gestures. A persistent lip protrusion gesture that is part of the representation of the first vowel overlaps the gestures of the following vowel, which surfaces as rounded as a result. The time course of lip protrusion below the gestural score indicates that the lips reach their target protruded state and remain there throughout the word. This is the basic representation of harmony within this model: the result of a single, uninterrupted harmonizing gesture with an extended activation period. 
(8) Rounding harmony due to overlap by a persistent lip protrusion gesture

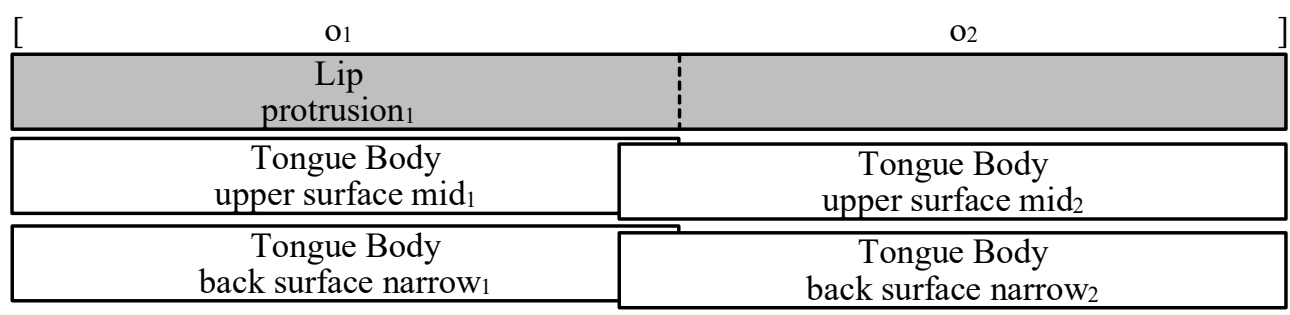

Resulting lip position:

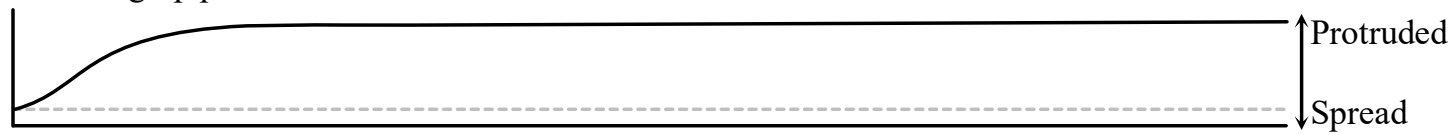

To account for transparency, the Gestural Harmony Model takes advantage of the fact that gestures are goal-based units; while they are specified for a target articulatory state, they may not necessarily achieve it successfully. In this model, transparent segments are considered a special type of undergoer. Rather than being skipped over by a harmony process, transparent segments are overlapped by a harmonizing gesture just as typical undergoer segments are. Transparency arises when gestural overlap results in antagonism, a situation in which two concurrently active gestures have opposing target articulatory states. Examples of antagonistic pairs of gestures include concurrently active gestures for lip protrusion and lip spreading, velum opening and velum closure, and narrow and wide constrictions at the upper surface of the vocal tract.

Because the concurrent achievement of two conflicting target articulatory states is not possible, antagonistic gestures are essentially in competition with one another for control of the vocal tract. The outcome of the competition between antagonistic gestures is determined by the relative strengths that are specified for each gesture. According to the Task Dynamic Model of speech production (Saltzman \& Munhall 1989, Fowler \& Saltzman 1993), when gestural antagonism occurs, it is resolved by blending the competing target articulatory states of these gestures to create an intermediate target state that holds during the period of their concurrent activation. This blended target state is the weighted average of two gestures' individual target articulatory states, and the weighting in this averaging function is contributed by the gestures' strength parameters, denoted $\alpha$. The gestural blending function is provided in (9).

(9) Gestural blending function in the Task Dynamic Model of speech production

$$
\text { Blended Target }=\frac{\text { Target }_{1} \cdot \alpha_{1}+\text { Target }_{2} \cdot \alpha_{2}}{\alpha_{1}+\alpha_{2}}
$$

To illustrate transparency via gestural blending with another case of rounding harmony, the figure in (10) contains the gestural score for an [o-i-o] sequence. The persistent lip protrusion gesture of the first [o] overlaps the gestures of all other vowels. The third vowel in the sequence surfaces as rounded [o] due to this overlap, while the medial high front /i/, also overlapped by the lip protrusion gesture, surfaces as [i] rather than $[\mathrm{y}]$. This is due to the representation of /i/ including an antagonistic lip spreading gesture in addition to its tongue body gestures. This lip spreading gesture is specified for a relatively high gestural strength; in this example, its strength is ten times the strength of the harmonizing gesture. This allows the lip spreading gesture to counteract the effect of the harmonizing lip protrusion gesture upon the vocal tract during the period of their concurrent activation. 
(10) Transparency due to relatively strong antagonistic lip spreading gesture active during production of relatively weak harmonizing lip protrusion gesture

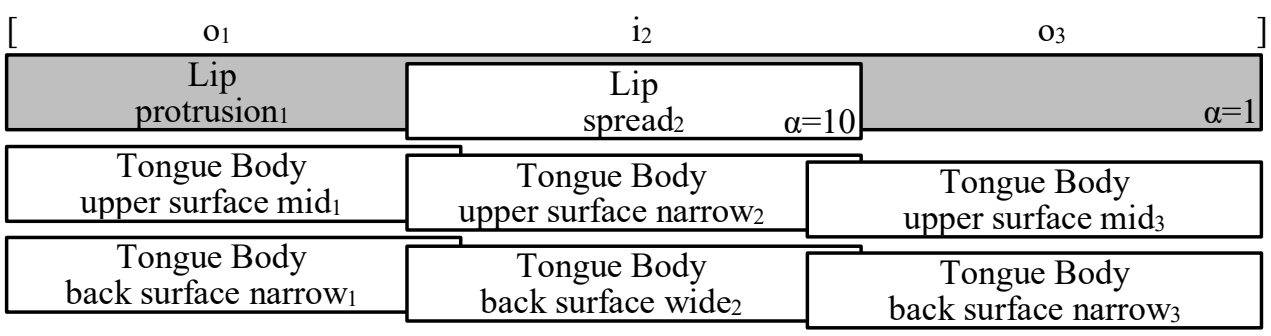

Resulting lip position:

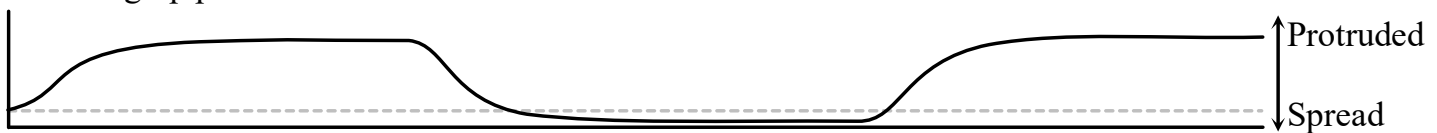

There are several advantages to this representation of harmony and transparency. First, it correctly predicts which segments may be transparent within a given type of harmony: those that include a gesture that is antagonistic to a harmonizing gesture. Smith $(2016,2018)$ claims that this is important for typological reasons; in rounding harmony and nasal harmony, the sets of crosslinguistically attested transparent segments is limited to precisely those classes of segments that instrumental evidence suggests include gestures that are antagonistic to a harmonizing gesture. In addition, this model provides a representation of harmony in which spreading is completely local. A harmony process does not skip over segments, even those that surface as transparent.

The full transparency of high front [i] to rounding harmony in (10) is dependent on the strength of its antagonistic lip spreading gesture being much greater than that of the harmonizing lip protrusion gesture, with an example strength ratio of 10-to-1. However, because gestural strength is defined numerically, it is not limited to simply categorizing gestures as either 'strong' or 'weak.' As a result, there is no restriction within the Gestural Harmony Model dictating that for a given pair of overlapped gestures, one must be much stronger than the other. Another possible scenario predicted by the model is one in which a harmonizing gesture and an overlapped antagonistic gesture have similar or identical strengths. When such a configuration arises, it predicts a case of blending resulting in partial transparency and partial undergoing of harmony, as in (11).

(11) Partial transparency resulting from equal strengths of harmonizing and antagonistic gestures

\begin{tabular}{|c|c|}
\hline $\begin{array}{c}\text { Harmonizing } \\
\text { Gesture }\end{array}$ & $\begin{array}{c}\text { Antagonistic } \\
\text { Gesture }\end{array}$ \\
\end{tabular}

Resulting state of vocal tract for some variable:

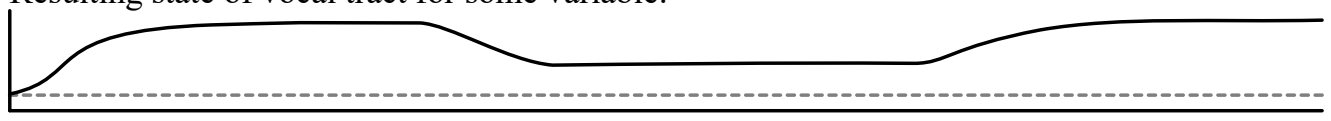

I propose that partial height harmony represents just such a case of partial transparency. The following section outlines an analysis of the partial height harmony evident in Servigliano Italian metaphony within the Gestural Harmony Model.

\section{Analysis}

Recall from section 1 that Servigliano Italian exhibits a case of partial, stepwise vowel raising harmony. In this harmony pattern, high-mid undergoers fully assimilate to the height of high vowel triggers of harmony, while low-mid vowels only partially assimilate to the trigger height. I propose that this pattern be treated as a case of partial transparency to harmony, and analyzed as the result of gestural blending within the Gestural Harmony Model. In contrast with full transparency, in which the antagonistic gesture of 
a transparent segment is much stronger than the harmonizing gesture, partial height harmony represents a case in which the two blended gestures are of roughly equal strengths.

This analysis relies on blending of the target constriction degrees of tongue body upper surface gestures for vowels of different heights. Each of the four vowel heights observed in Servigliano Italian is represented by a tongue body upper surface gesture with one of four possible constriction degrees: narrow, narrow-mid, wide-mid, and wide. I analyze the vowel raising harmony in Servigliano Italian as the result of overlap by an anticipatory upper surface gesture with narrow constriction degree that is part of the representation of suffix high vowels $/ \mathrm{i} /$ and $/ \mathrm{u} /$. Under this analysis, vowels that are specified for a widemid constriction and that appear to be partially transparent to this vowel raising harmony are able to partially resist the raising effect of the triggering narrow vowel gesture because they are of equal blending strengths. The weaker high-mid vowels, on the other hand, surface as high rather than resisting raising, suggesting that they have a relatively lower blending strength. This is the subject of section 3.1. In section 3.2, I also outline a possible interpretation of the lack of raising of the low vowel as a case of full transparency due to high gestural strength.

3.1 Mid vowel raising In addition to being specified for a target constriction degree, the tongue body upper surface gesture of each vowel is specified for a strength value that is used to calculate the outcome of blending between antagonistic gestures. Rather than simply referring to 'relatively strong' and 'relatively weak' gestures, this analysis provides a set of precise gestural blending strengths that produce the desired surface vowel constriction degrees for Servigliano Italian metaphony when input to the blending function in (9). The table in (12) provides the non-low vowels with target constriction degrees for their upper surface gestures, as well as proposed strength values. The precise strength values proposed here are not crucial; rather, it is the strength ratios between gestures that are important to the analysis.

(12) Target constriction degrees and gestural blending strengths for Servigliano Italian upper surface gestures for high and mid vowels

\begin{tabular}{ccc}
\hline Vowel & Target Constriction Degree & Strength $(\alpha)$ \\
\hline$/ \mathrm{i} /, / \mathrm{u} /$ & $4 \mathrm{~mm}$ & 10 \\
$/ \mathrm{e} /, / \mathrm{o} /$ & $8 \mathrm{~mm}$ & 1 \\
$/ \varepsilon /, / \mathrm{o} /$ & $12 \mathrm{~mm}$ & 10 \\
\hline
\end{tabular}

As a result of gestural blending, narrow-mid vowels /e/ and /o/ fully undergo harmony and surface as raised. This is achieved by specifying that /e/ and /o/ have a much lower strength than the high vowels /i/ and $/ \mathrm{u} /$. As shown in (13), the gestural blending function produces a blended target constriction degree of $4.36 \mathrm{~mm}$, very similar to the proposed $4 \mathrm{~mm}$ constriction degree of a narrow vowel trigger.

(13) Gestural blending of narrow and narrow-mid vowels

$$
4.36 \mathrm{~mm}=\frac{\overbrace{4 \mathrm{~mm} \cdot 10}^{/ \mathrm{i} /, / \mathrm{u} /}+\overbrace{8 \mathrm{~mm} \cdot 1}^{/ \mathrm{e} /, / \mathrm{o} /}}{10+1}
$$

This blending outcome is illustrated by the gestural score in (14) for the upper surface gestures of a word such as [kríd-i] 'you believe,' in which the first [i] is the result of raising of an underlying /e/. The accompanying time course of tongue body height indicates that the underlying narrow-mid vowel /e/ fully undergoes harmony and surfaces as [i] when it is overlapped by the anticipatory upper surface gesture of a following, stronger narrow vowel [i]. 
(14) Narrow-mid to narrow vowel raising

\begin{tabular}{|c|c|c|c|}
\hline$i_{1}$ & & $\mathrm{i}_{2}$ & \\
\hline $\begin{array}{c}\text { Tongue Body } \\
\text { upper surface narrow-mid }\end{array}$ & $\alpha=1$ & $\begin{array}{l}\text { Tongue Body } \\
\text { upper surface narrow } 2 \\
\end{array}$ & $\alpha=10$ \\
\hline
\end{tabular}

Resulting tongue body height:

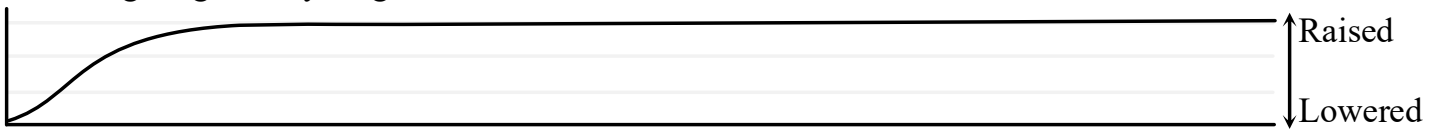

Wide-mid vowels, on the other hand, resist fully undergoing harmony, but also do not surface as fully transparent to harmony. This partial transparency is produced by providing narrow triggers $/ \mathrm{i} / \mathrm{and} / \mathrm{u} /$ and wide-mid undergoers $/ \varepsilon /$ and $/ \mathrm{o} /$ with equal strengths. The blending function does not favor either target articulatory state over the other, but instead returns a target constriction degree of $8 \mathrm{~mm}$, as in (15). This is intermediate between the two antagonistic target constriction degrees and consistent with that of the underlying narrow-mid vowels.

(15) Gestural blending of narrow and wide-mid vowels

$$
8 \mathrm{~mm}=\frac{\overbrace{4 \mathrm{~mm} \cdot 10}^{/ \mathrm{i} /, / \mathrm{u} /}+\overbrace{12 \mathrm{~mm} \cdot 10}^{/ \varepsilon /, / \mathrm{o} /}}{10+10}
$$

As a result of this blending calculation, $/ \varepsilon /$ and $/ \mathrm{J}$ only partially undergo harmony and surface as partially raised. The gestural score in (16) consists of the upper surface gestures of a word such as [sgwéts-u] 'suspicious (m. sg.),' in which the [e] is the result of raising. The time course of tongue body height shows that the underlyingly wide-mid vowel $/ \varepsilon /$ only partially undergoes harmony, surfacing as $[\mathrm{e}]$ when it is overlapped by the upper surface gesture of a following narrow vowel $[u]$.

(16) Wide-mid to narrow-mid vowel raising

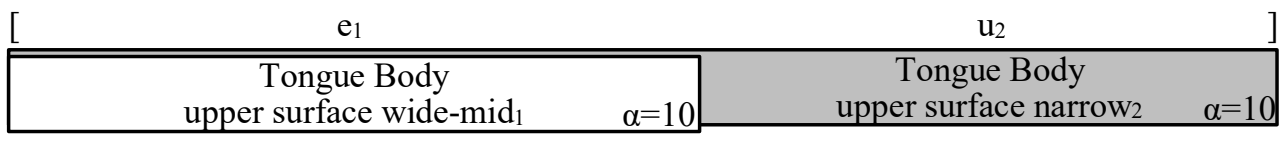

Resulting tongue body height:

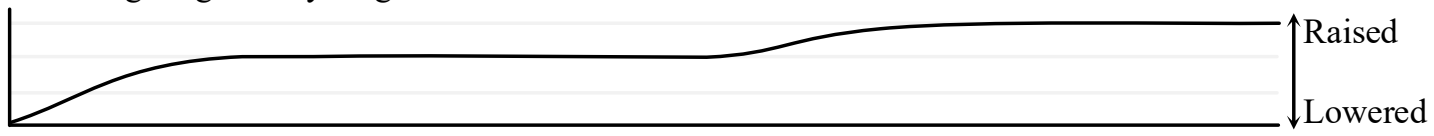

Provided with the proper strength values for vocalic upper surface gestures of different constriction degrees, the mechanism of gestural blending is successfully recruited by the Gestural Harmony Model to produce blended target constriction degrees that are consistent with the vowel raising pattern in Servigliano Italian. Due to overlap by the anticipatory upper surface gesture of a narrow vowel, underlyingly narrowmid vowels are fully raised and surface as narrow, while underlyingly wide-mid vowels only partially undergo raising to surface as narrow-mid. Both of these outcomes arise from their upper surface gestures' intrinsic specifications for constriction degree and blending strength.

3.2 The low vowel Recall that in Servigliano Italian the low vowel /a/ does not undergo raising, surfacing faithfully as [a] before high and non-high vowels alike. There are two possible interpretations of this seeming lack of participation in the metaphony process. One is that /a/ blocks harmony in Servigliano Italian and is not raised because it is not overlapped by the tongue body upper surface gesture of narrow vowels $/ \mathrm{i} / \mathrm{and} / \mathrm{u} /$, the triggers of harmony. (On the representation of blocking in the Gestural Harmony 
Model, see Smith $(2016,2018)$.) Another interpretation is that /a/ is overlapped by the upper surface gestures of triggering narrow vowels, but is fully transparent to harmony and does not raise because it is substantially stronger than the harmonizing gesture overlapping it.

Because metaphony is a bounded harmony process that does not extend beyond the stressed vowel, there is no way to definitively tell which of these interpretations of the low vowel's lack of raising is correct. However, there is indirect evidence that /a/ is transparent to metaphony. In Servigliano Italian, /a/ is transparent to a separate process of pre-tonic vowel raising harmony that is triggered by stressed high vowels. This is exemplified in (17c-d), in which mid vowel raising is triggered by a stressed high vowel. In each of these forms, the first mid vowel raises to surface as high despite the presence of an intervening [a].

(17) a. [bokalétt-a] (no gloss)

b. [bokkkal-ó] 'foolish (m. sg.)' c. [bukkalítt-u] (no gloss)

d. [bukkkal-ú] 'foolish (m. pl.)'

Under the assumption that /a/ is transparent to metaphony as well as pre-tonic vowel raising, I analyze this as a case of full transparency due to high gestural strength relative to the trigger of harmony. The blending function in (18) produces a wide target constriction degree of $15.43 \mathrm{~mm}$, strongly favoring the intrinsic constriction degree of the low vowel $(16 \mathrm{~mm})$ rather than that of the narrow vowel when their two gestures are blended. This is due to the much greater strength $(\alpha=200)$ of the wide vowel's upper surface gesture.

(18) Gestural blending of narrow and wide vowels

$$
15.43 \mathrm{~mm}=\frac{\overbrace{4 \mathrm{~mm} \cdot 10}^{/ \mathrm{i} /, / \mathrm{u} /}+\overbrace{16 \mathrm{~mm} \cdot 10}^{/ \mathrm{a} /}}{10+200}
$$

As a result of its high gestural strength, /a/ is not raised when its wide upper surface gesture is overlapped by an anticipatory narrow upper surface gesture from high vowels $/ \mathrm{i} /$ or $/ \mathrm{u} /$. This is illustrated by the gestural score and time course of tongue body height in (19).

(19) No raising of wide vowel due to high gestural strength

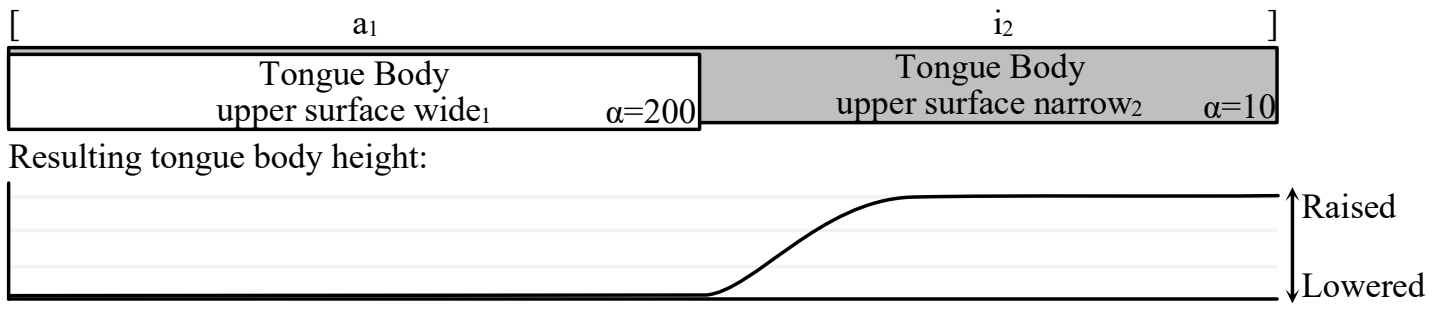

The Gestural Harmony Model successfully accounts not only for the full and partial raising of the mid vowels in Servigliano Italian, but also for the lack of raising of wide /a/. Fully undergoing harmony as well as remaining either partially or fully transparent to it are all accomplished via a single theoretical mechanism: gestural blending.

\section{Alternative: Synchronic Chain Shifts in Feature-Based Phonology}

The analysis of partial height harmony as partial transparency fulfills a strong prediction made by the Gestural Harmony Model: that intermediate strength values produce cases of partial undergoing and partial resistance due to overlap by a harmonizing gesture. In addition to fulfilling this prediction, the gestural analysis also provides solutions to issues that arise within featural analyses of partial height harmony. Chief among these is the stepwise nature of some harmony patterns forming apparent synchronic chain shifts with respect to vowel height. For instance, in Servigliano Italian, metaphony produces raising patterns 
$\varepsilon \rightarrow \mathrm{e} \rightarrow \mathrm{i}$ and $\mathrm{o} \rightarrow \mathrm{o} \rightarrow \mathrm{u}$. Such chain shifts present some difficulty for output-oriented frameworks such as Optimality Theory (Prince \& Smolensky 1993/2004) and Harmonic Grammar (Legendre, Miyata, \& Smolensky 1990, Smolensky \& Legendre 2006), due to their apparent derivational opacity. In their base forms, these frameworks are unable to account for a mapping like $\varepsilon \rightarrow \mathrm{e} \rightarrow \mathrm{i}$, in which [e] is permitted to surface when it is derived from $/ \varepsilon /$ (as opposed to mapping input $/ \varepsilon /$ to output [i]), but not when it surfaces faithfully from underlying /e/.

Kirchner (1996) and Moreton \& Smolensky (2002) propose that chain shifts can be generated in Optimality Theory via the conjunction of faithfulness constraints. In constraint conjunction (Smolensky 1993), a new constraint is added to CON by combining two simple constraints. This conjoined constraint is only violated by a candidate that contains violations of both composite constraints within some specified domain. With conjoined faithfulness constraints, a phonological grammar is able to restrict an input-output mapping such that it may be unfaithful, but not too unfaithful, thereby producing stepwise chain shift.

In the case of Servigliano Italian, the necessary conjoined constraint prevents a potential undergoer of harmony from altering its underlying values for more than one height feature, producing partial rather than full raising of the low-mid vowels. This is precisely the approach taken by Walker (2011) in analyzing Servigliano Italian metaphony. In her analysis, the conjoined faithfulness constraint IDENT(high)\&IDENT(ATR) is ranked above the harmony-driving LICENSE([Height],ó), which is satisfied by the spread of a high post-tonic trigger vowel's height features ([+high], [-low], and [+ATR]) onto the vowel of a stressed syllable. This approach is illustrated by the tableau in (20).

(20) Servigliano Italian partial height harmony via constraint conjunction

\begin{tabular}{|c|c|c|c|c|}
\hline Input: / $\varepsilon-\mathrm{i} /$ & IDENT(high)\&IDENT(ATR) & LiCENSE([Height],ó) & IDENT(high) & IDENT(ATR) \\
\hline a. $[\mathrm{i}-\mathrm{i}]$ & $* !$ & & * & * \\
\hline b. $\quad[\mathrm{e}-\mathrm{i}]$ & & * & & * \\
\hline c. $[\varepsilon-i]$ & & $* * !$ & & \\
\hline Input: /e-i/ & IDENT(high)\&IDENT(ATR) & LiCENSE([Height],ó) & IDENT(high) & IDENT(ATR) \\
\hline d. $\quad[\mathrm{i}-\mathrm{i}]$ & & & * & \\
\hline e. $[\mathrm{e}-\mathrm{i}]$ & & $* !$ & & \\
\hline
\end{tabular}

In (20), the input vowel sequence / $\varepsilon$-i/ surfaces as winning candidate (b) [e-i], which satisfies the harmonydriving LICENSE to a greater degree than the faithful candidate (c) $[\varepsilon-\mathrm{i}]$, despite incurring a violation of IDENT(ATR). Candidate (a) [i-i] fully satisfies LICENSE due to the full assimilation between trigger and undergoer, but in doing so fatally violates the conjoined faithfulness constraint by altering the undergoer's [ \pm high] and $[ \pm \mathrm{ATR}]$ specifications. For the input sequence /e-i/, candidate (d) [i-i] is the winner, as it fully satisfies LICENSE by violating only IDENT(high) and not the conjoined faithfulness constraint.

The tableau in (20) demonstrates that the conjunction of faithfulness constraints is able to successfully account for synchronic chain shifts. However, the admission of constraint conjunction as a grammatical mechanism within Optimality Theory has been called into question. Itô \& Mester (1998), Fukazawa \& Lombardi (2003), Pater (2009), and Potts, Pater, Jesney, Bhatt, \& Becker (2010) report that many independently motivated individual constraints produce unattested patterns when conjoined. For instance, Fukazawa \& Lombardi show that when conjoined, the constraints NOCODA and *VOICE predict an unattested phonological system that repairs voiced codas.

Another common method of modeling constraint interaction is via constraint ganging in Harmonic Grammar. In constraint ganging, one candidate's violation of two lower-weighted constraints is greater than another candidate's violation of a single higher-weighted constraint. This produces many of the same phonological patterns as constraint conjunction, but is often argued to be a superior approach to cumulative constraint interaction due to its typological restrictiveness. However, Albright, Magri, \& Michaels (2008), Farris-Trimble (2008), and Magri (2018) show that constraint ganging in Harmonic Grammar is unable to produce chain shifts without significantly altering the grammar's EVAL mechanism. This is illustrated by the tableau in (21) for the input $/ \varepsilon-\mathrm{i} /$, using the same constraints used to illustrate constraint conjunction in (20). 
(21) Failed constraint ganging for Servigliano Italian metaphony

\begin{tabular}{|r||c|c|c|c|}
\hline Input: $/ \varepsilon-\mathrm{i} /$ & $\begin{array}{c}\text { LICENSE([Height], б) } \\
\mathrm{w}=3\end{array}$ & $\begin{array}{c}\text { IDENT(high) } \\
\mathrm{w}=2\end{array}$ & $\begin{array}{c}\text { IDENT(ATR) } \\
\mathrm{w}=2\end{array}$ & $\mathcal{H}$ \\
\hline \hline a. $[\mathrm{i}-\mathrm{i}]$ & & -1 & -1 & -4 \\
\hline b. $[\mathrm{e}-\mathrm{i}]$ & -1 & & -1 & -5 \\
\hline c. $[\varepsilon-\mathrm{i}]$ & -2 & & & -6 \\
\hline
\end{tabular}

In (21), the constraint weights are set such that LICENSE outweighs each IDENT constraint individually, but violation of both IDENT constraints outweighs violation of LICENSE. However, this configuration does not produce the desired chain shift. Rather than partial raising candidate (b) [e-i] being chosen as the winner, this constraint weighting selects full raising candidate (a) [i-i]. Constraint ganging does not arise due to the absence of a necessary asymmetric tradeoff; the violation of both IDENT constraints by candidate (a) does not outweigh the violation of LICENSE by candidate (b) because candidate (b) also violates IDENT(ATR). This result arises independent of the precise constraint used to drive harmony as well as the weights chosen for these constraints.

The characterization of stepwise, partial height harmony is quite different in the analysis within the Gestural Harmony Model. In Servigliano Italian, gestural blending between trigger and undergoer vowel gestures produces the effect of vowel raising. However, the individual gestures of each vowel are still present and unaltered in the gestural score of the surface phonological form, as shown in (22).

(22) Comparing the gestural representations of underlying and derived [e-i] sequences

(a) An [e-i] sequence derived from an underlying $/ \varepsilon-1 /$ sequence via gestural overlap

\begin{tabular}{|c|c|}
\hline$\varepsilon_{1}$ & $\mathrm{i}_{2}$ \\
\hline $\mathrm{e}_{1}$ & $\mathrm{i}_{2}$ \\
\hline $\begin{array}{c}\text { Tongue Body } \\
\text { upper surface wide-mid }\end{array}$ & $\begin{array}{c}\text { Tongue Body } \\
\text { upper surface narrow2 }\end{array}$ \\
\hline
\end{tabular}

(b) An [e-i] sequence surfacing faithfully from an underlying /e-i/ sequence

\begin{tabular}{|c|c|}
\hline $\mathrm{e}_{1}$ & $\mathrm{i}_{2}$ \\
\hline $\mathrm{e}_{1}$ & $\mathrm{i}_{2}$ \\
\hline $\begin{array}{c}\text { Tongue Body } \\
\text { upper surface narrow-mid } 1\end{array}$ & $\begin{array}{c}\text { Tongue Body } \\
\text { upper surface narrow2 }\end{array}$ \\
\hline
\end{tabular}

While an [e] derived by gestural overlap and blending between $/ \varepsilon /$ and $/ \mathrm{i} /$, as in (22a), and an [e] that surfaces faithfully from an underlying /e/, as in (22b), may be articulatorily and acoustically the same, their gestural makeups are different in both the phonological input and output. Such an approach is unavailable to featural representations of this process. A surface [e] that is derived from $/ \varepsilon /$ is featurally indistinguishable from one that surfaces faithfully from /e/, necessitating reliance on grammatical mechanisms such as cumulative constraint interaction to account for apparent derivational opacity. In the Gestural Harmony Model, on the other hand, partial stepwise harmony need not be modeled as an opaque process, despite its appearance as a synchronic chain shift. As a result, the model is able to avoid the difficulties of generating opaque patterns that arise in featural analyses of partial height harmony within either the Optimality Theory or Harmonic Grammar frameworks.

\section{Conclusion}

In this paper, I have provided an analysis of partial, stepwise height harmony in Servigliano Italian as an instance of partial transparency within the Gestural Harmony Model. This analysis relies on the theoretical mechanism of blending of gestures with antagonistic target states. By relying on a specification 
of strength that is richer than a simple distinction between 'strong' and 'weak,' this single mechanism is able to produce several different outcomes of harmony via gestural overlap in Servigliano Italian: full raising by high-mid vowels, partial raising by low-mid vowels, and total resistance to harmony by low vowels. In addition to fulfilling a prediction of the Gestural Harmony Model, the analysis of partial height harmony as partial transparency provides a valuable new perspective on patterns that have the appearance of synchronic chain shifts, and avoids issues that arise from many featural analyses of such apparently opaque patterns.

\section{References}

Albright, Adam, Magri, Giorgio, \& Michaels, Jennifer (2008) Modeling Doubly Marked Lags with a Split Additive Model. In H. Chan, H. Jacob, \& E. Kapia (Eds.), Proceedings of the 32nd Annual Boston University Conference on Language Development (pp. 36-47). Cascadilla Press.

Browman, Catherine, \& Goldstein, Louis (1986) Towards an Articulatory Phonology. Phonology Yearbook, 3, 219252.

Browman, Catherine, \& Goldstein, Louis (1989) Articulatory gestures as phonological units. Phonology, 6, $201-251$.

Camilli, Amerindo (1929) Il dialetto di Servigliano. Archivum Romanicum, 13, 220-271.

Farris-Trimble, Ashley (2008) Cumulative Faithfulness Effects in Phonology. Ph.D. Dissertation, Indiana University.

Fowler, Carol, \& Saltzman, Elliot (1993) Coordination and Coarticulation in Speech Production. Language and Speech, 36, 171-195.

Fukazawa, Haruka, \& Lombardi, Linda (2003) Complex constraints and linguistic typology in Optimality Theory. The Linguistic Review, 20, 195-215.

Itô, Junko, \& Mester, Armin (1998) Markedness and word structure: OCP effects in Japanese. Unpublished ms., University of California Santa Cruz.

Kaze, Jeffery (1989) Metaphony in Italian and Spanish dialects revisited. Ph.D. Dissertation, University of Illinois at Urbana-Champaign.

Kirchner, Robert (1996) Synchronic Chain Shifts in Optimality Theory. Linguistic Inquiry, 27, 341-350.

Legendre, Géraldine, Miyata, Yoshiro, \& Smolensky, Paul (1990) Can Connectionism Contribute to Syntax? Harmonic Grammar, with an Application. In Proceedings of the 30th Meeting of the Chicago Linguistic Society.

Magri, Giorgio (2018) Idempotency, Output-Drivenness and the Faithfulness Triangle Inequality: Some Consequences of McCarthy's (2003) Categoricity Generalization. Journal of Logic, Language and Information, 27, 1-60.

Mascaró, Joan (2011) An analysis of stress-dependent harmony in Servigliano. Probus, 23, 21-55.

Moreton, Elliott, \& Smolensky, Paul (2002) Typological consequences of local constraint conjunction. In L. Mikkelsen \& C. Potts (Eds.), Proceedings of the 21st West Coast Conference on Formal Linguistics (pp. 306-319). Cascadilla Press.

Nibert, Holly (1998) Processes of vowel harmony in the Servigliano dialect of Italian: A comparison of two non-linear proposals for the representation of vowel height. Probus, 10, 67-102.

Pater, Joe (2009) Review: Paul Smolensky and Géraldine Legendre, The harmonic mind: from neural computation to optimality-theoretic grammar. Phonology, 26, 217-226.

Potts, Christopher, Pater, Joe, Jesney, Karen, Bhatt, Rajesh, \& Becker, Michael (2010) Harmonic Grammar with linear programming: from linear systems to linguistic typology. Phonology, 27, 77-117.

Prince, Alan, \& Smolensky, Paul (1993) Optimality Theory: Constraint Interaction in Generative Grammar. Published 2004 by Blackwell Publishing.

Saltzman, Elliot, \& Munhall, Kevin (1989) A Dynamical Approach to Gestural Patterning in Speech Production. Ecological Psychology, 1, 333-382.

Smith, Caitlin (2016) A gestural account of neutral segment asymmetries in harmony. In G. Hansson, A. FarrisTrimble, K. McMullin, \& D. Pulleyblank (Eds.), Proceedings of the 2015 Annual Meeting on Phonology. Linguistic Society of America.

Smith, Caitlin (2017a) Harmony Triggering as a Segmental Property. In A. Lamont \& K. Tetzloff (Eds.), Proceedings of the 47th Annual Meeting of the North East Linguistic Society. Graduate Linguistics Student Association.

Smith, Caitlin (2017b) Harmony Triggering as a Contrastive Property of Segments. In K. Jesney, C. O'Hara, C. Smith, \& R. Walker (Eds.), Proceedings of the 2016 Annual Meeting on Phonology. Linguistic Society of America.

Smith, Caitlin (2018) Harmony in Gestural Phonology. Ph.D. Dissertation, University of Southern California.

Smolensky, Paul (1993) Harmony, Markedness, and Phonological Activity. Presentation at Rutgers Optimality Workshop I (ROW-I).

Smolensky, Paul, \& Legendre, Géraldine (2006) The Harmonic Mind: From Neural Computation to OptimalityTheoretic Grammar. The MIT Press.

Walker, Rachel (2011) Vowel Patterns in Language. Cambridge University Press. 